\title{
Thyroid-stimulating hormone levels in newborns and early life exposure to endocrine-disrupting chemicals: analysis of three European mother-child cohorts
}

\author{
Marijke de Cock ${ }^{1}$, Michiel R. de Boer ${ }^{2}$, Eva Govarts ${ }^{3}$, Nina Iszatt ${ }^{4}$, Lubica Palkovicova ${ }^{5}$, Marja H. Lamoree ${ }^{6}$, Greet Schoeters ${ }^{7}$, \\ Merete Eggesb $\varnothing^{4}$, Tomas Trnovec ${ }^{5}$, Juliette Legler ${ }^{8}$ and Margot van de Bor ${ }^{1}$
}

BACKGROUND: Various studies have reported interactions between thyroid hormones and early life chemical exposure. Our objective was to analyze the associations between markers of endocrine-disrupting chemical exposure and thyroid function in newborns, determined through heel prick blood spots.

METHODS: Three European mother-child cohorts (FLEHSIBelgium, HUMIS-Norway, and the PCB cohort-Slovakia. Total $n=1,784$ ) were pooled for the purpose of this study. Data on thyroid-stimulating hormone (TSH) were obtained from national neonatal screening registries, and samples of cord plasma and/or breast milk were collected to determine exposure to various chemicals. Multiple regression models were composed with exposure and cohort as fixed factors, and adjustments were made for a priori defined covariates.

RESULTS: Median TSH concentrations were 1, 1.10, and 2.76 $\mathrm{mU} / \mathrm{l}$ for the Belgian, Norwegian, and Slovak cohorts, respectively. For polychlorinated biphenyl (PCB)-153 and dichlorodiphenyldichloroethylene ( $\left.p, p^{\prime}-D D E\right)$, children in the third exposure quartile had a 12-15\% lower TSH at birth. Results remained unchanged after additional adjustment for birth weight and gestational weight gain. No effect on TSH was observed for the other compounds.

CONCLUSION: Early life exposure to PCB-153 and p,p'-DDE impacts newborn TSH levels. Higher exposure levels were associated with 12-15\% lower TSH levels.

$T$ he use of synthetic pesticides in agriculture grew rapidly after the Second World War, and although certain pesticides are now banned their presence can still be detected in the environment. The Centers for Disease Control and Prevention (CDC) released a report on human biomonitoring in the United States, showing that a variety of pesticides could be detected in $90-100 \%$ of participants (1). Furthermore, the presence of several groups of chemicals (polychlorinated biphenyls (PCBs), organochlorine pesticides, perfluoroalkyl substances (PFASs), brominated flame retardants (PBDEs), phthalates, and polycyclic aromatic hydrocarbons (PAHs)) could also be detected in $99-100 \%$ of pregnant women (2). As several of these chemicals have been detected in cord blood (3) and amniotic fluid (4), it is clear that the placenta does not protect the fetus from exposure.

Exposure to chemicals during the prenatal period has been associated with adverse health effects in children as well as in adults (5). Increased risk for higher body mass index (BMI) during childhood was found to be related to prenatal exposure to PCBs (3), p, p'-dichlorodiphenyldichloroethene (p,p'-DDE) (6), hexachlorobenzene (HCB) (7), and dioxin/furans (8). Prenatal exposure to perfluorinated alkyl acids was associated with an increased risk for a higher BMI and larger waist circumference in 20-year-old females (9). Furthermore, PCBs, $\mathrm{HCB}$, and chlorpyrifos have also been found to be associated with behavioral disorders, including attention-deficit hyperactivity disorder and autism spectrum disorders (10).

The toxic effects of chemicals may be attributed to various mechanisms, including interference with hormonal function, which may have long-lasting consequences, especially during periods of great developmental plasticity (11). Various experimental studies have reported interactions between endocrine-disrupting chemical (EDC) exposure and plasma thyroid hormones (TH) (12). The thyroid gland produces thyroxine (T4) and triiodothyronine (T3), which are regulated through a negative feedback loop with thyroid-stimulating hormone (TSH) excreted by the pituitary gland. The fetus depends on the mother for supply of thyroid hormones until the fetal thyroid gland reaches maturity, which is around week 16 of gestation (13). As TH are involved in neurogenesis, neuronal migration, and myelination, they are essential for brain development (14). Prenatal exposure to the $\mathrm{PCB}$ metabolite $4-\mathrm{OH}-2,3,3^{\prime}, 4^{\prime}, 5$-pentachlorobiphenyl resulted in lower total T4 levels in both male and female rats at postnatal

\footnotetext{
'Department of Environment and Health, Vrije Universiteit, Amsterdam, The Netherlands; ${ }^{2}$ Department of Health Sciences, Vrije Universiteit, Amsterdam, The Netherlands; ${ }^{3}$ Unit Environmental Risk and Health, Flemish Institute for Technological Research (VITO), Mol, Belgium; ${ }^{4}$ Division of Epidemiology, Norwegian Institute of Public Health. Oslo, Norway; ${ }^{5}$ Department of Environmental Medicine, Faculty of Public Health, Slovak Medical University, Bratislava, Slovakia; ${ }^{6}$ Institute for Environmental Studies, Vrije Universiteit, Amsterdam, The Netherlands; ${ }^{7}$ Department of Biomedical Sciences, University of Antwerp, Antwerp, Belgium; Institute of Environment, Health and Societies, Brunel University, London, UK. Correspondence: Marijke de Cock (m.de.cock@vu.nl) 
Table 1. Characteristics of the study sample

\begin{tabular}{|c|c|c|c|c|c|c|}
\hline & \multicolumn{2}{|c|}{ FLEHSI } & \multicolumn{2}{|c|}{ HUMIS } & \multicolumn{2}{|c|}{ PCB cohort } \\
\hline & $n$ & & $n$ & & $n$ & \\
\hline Sex child (boy, \%) & 1,105 & 52.1 & 230 & 53.9 & 449 & 50.6 \\
\hline Birth weight $(g)$ & 1,105 & $3,400.7(450.5)$ & 230 & $3,635.4(499.4)$ & 449 & $3,315.1(497.8)$ \\
\hline Gestational age (weeks) & 1,105 & $39.3(1.3)$ & 228 & $40.2(1.6)$ & 446 & $39.7(1.1)$ \\
\hline Pre-pregnancy BMI mother $\left(\mathrm{kg} / \mathrm{m}^{2}\right)$ & 1,072 & $23.2(4.1)$ & 225 & $24.6(4.8)$ & 425 & $22(3.8)$ \\
\hline Maternal gestational weight gain (kg) & 540 & $14.4(5.6)$ & 224 & $14.6(5.8)$ & 307 & $13.8(4.6)$ \\
\hline Maternal age at birth (years) & 1,098 & $29.1(4.3)$ & 230 & $28.8(4.5)$ & 443 & $25.3(4.7)$ \\
\hline Nulliparous (yes, \%) & 1,105 & 60.5 & 230 & 48.3 & 447 & 45.4 \\
\hline Smoking during pregnancy (yes, \%) & 1,102 & 15.7 & 230 & 10.4 & 449 & 16 \\
\hline Alcohol during pregnancy (yes, \%) & 1,096 & 8.4 & 124 & 16.9 & 449 & 24.3 \\
\hline
\end{tabular}

Values are mean (SD) unless indicated otherwise.

day 4 (15). No effects were observed on TSH. Shi et al. (12) exposed zebrafish to $200 \mu \mathrm{g} / \mathrm{l}$ pefluorooctane sulfonate (PFOS) through the water and observed an upregulation of TSH gene expression. However, at higher dosage $(400 \mu \mathrm{g} / \mathrm{l})$ downregulation of the TSH gene was observed, which may be an indication of a non-linear dose-response relationship, a phenomenon frequently observed with EDCs (16). Observational studies have also reported associations between environmental exposures and TH. Cord blood PCB-153 was negatively associated with thyroid-binding globulin (TBG) in newborn Inuit children (17) and positively associated with TSH in a cohort of Spanish children (18). HCB, DDE, and perfluorooctanoic acid (PFOA) exposure has also been associated with cord blood TSH and T4 (19). Early life exposure to several PBDE congeners showed inverse associations with plasma T3 or free T3 levels (20). However, other studies have reported positive associations between exposure to PBDEs and both cord blood T3 and T4 (21), and some have observed no effect at all (22). Results on PCB-153, $\mathrm{p}, \mathrm{p}^{\prime}$-DDE, and HCB are also inconclusive, as several studies have reported no effect of exposure on plasma TH (22).

Otake et al. (23) observed an association between thyroid hormones measured in heel prick blood spots and PCBs measured in umbilical cord tissue. No association was observed between concentrations of total PCBs or individual congeners and either fT4 or TSH levels. However, total hydroxylated PCB (OH-PCB) metabolites and OH-PCB-187 were associated with higher fT4 levels. The use of heel prick blood is interesting because this matrix is available for all children in many countries where it is collected as a part of national screening programs. Furthermore, stress associated with delivery may influence TSH levels in cord blood (24). Heel prick blood is sampled a few days after birth, when the effects of delivery have started to level off.

To explore the association between markers of EDC exposure and thyroid function in newborns, we used TSH levels determined in heel prick blood from three birth cohorts participating in the OBELIX project (25). To our knowledge, this is the largest study conducted on this topic thus far, including on multiple types of chemicals such as polychlorinated biphenyls, organochlorine pesticides, flame retardants, and perfluorinated alkyl acids.

\section{METHODS}

\section{Description of Cohorts}

Three cohorts from across Europe, participating in the OBELIX project (25), and all including live-born singletons, were included (Table 1). Mother-child pairs from the FLEHSI cohort, Belgium, were recruited between 2002 and 2004. Data on EDC exposure levels in cord plasma and TSH levels from the heel prick test were available on 1,105 out of 1,196 mother-child pairs. The HUMIS cohort (Norway, $n=230$ out of 2,606, 2002-2006) measured exposure levels in breast milk. Data on chemical exposures of interest in this project were available for 396 subjects at the time of analysis. TSH values were accessible only for 239 of them, who were those born after the TSH values had been registered in a central registry. The HUMIS cohort also included oversampled small for gestational age and preterm cases, which were excluded from the current study, leaving a total of 230 mother-child pairs. The PCB cohort (Slovakia, 20022004) measured exposure in maternal blood, cord serum, or breast milk. Data on exposure levels in cord serum or breast milk, as well as TSH levels, were available on 449 out of 1,134 mother-child pairs. All cohorts targeted the general population. All cohorts were approved by appropriate national ethics committees (FLEHSI: Medical Ethics Committee University of Antwerp; HUMIS: Norwegian Data Inspectorate and Regional Ethics Committee for Medical Research; PCB cohort: Institutional Review Board at the Slovak Medical University) and written informed consent was obtained from every mother. Details of enrollment and exclusion criteria for each cohort are given in Supplementary Table S1.

\section{TSH}

Heel prick screening after birth that is focused on detecting inborn errors of metabolism, such as phenolketonuria, is a common methodology of screening in many countries, including the countries to which the cohorts of this study belonged. Within 4-6 days of birth, blood samples were collected on a filter paper from each newborn through heel puncture, which were then analyzed for TSH. TSH was analyzed by means of Autodelfia (limit of detection [LOD]: $1 \mathrm{mU} / \mathrm{l})(26)$, except for the Slovak samples, which were analyzed by the immunoluminometric quantitive method (analytical sensitivity: 5 mIU/l; neo TSH ILMA Kit, fi Immunotech Beckman Coulter, Bratislava, Slovakia).

\section{Exposure to EDCs}

Exposure was assessed in either cord blood (FLEHSI and PCB cohort) or breast milk (HUMIS and PCB cohort). Breast milk in HUMIS was sampled $\sim 1$ month after delivery, and each participant was asked to collect a pooled sample over eight consecutive days. 


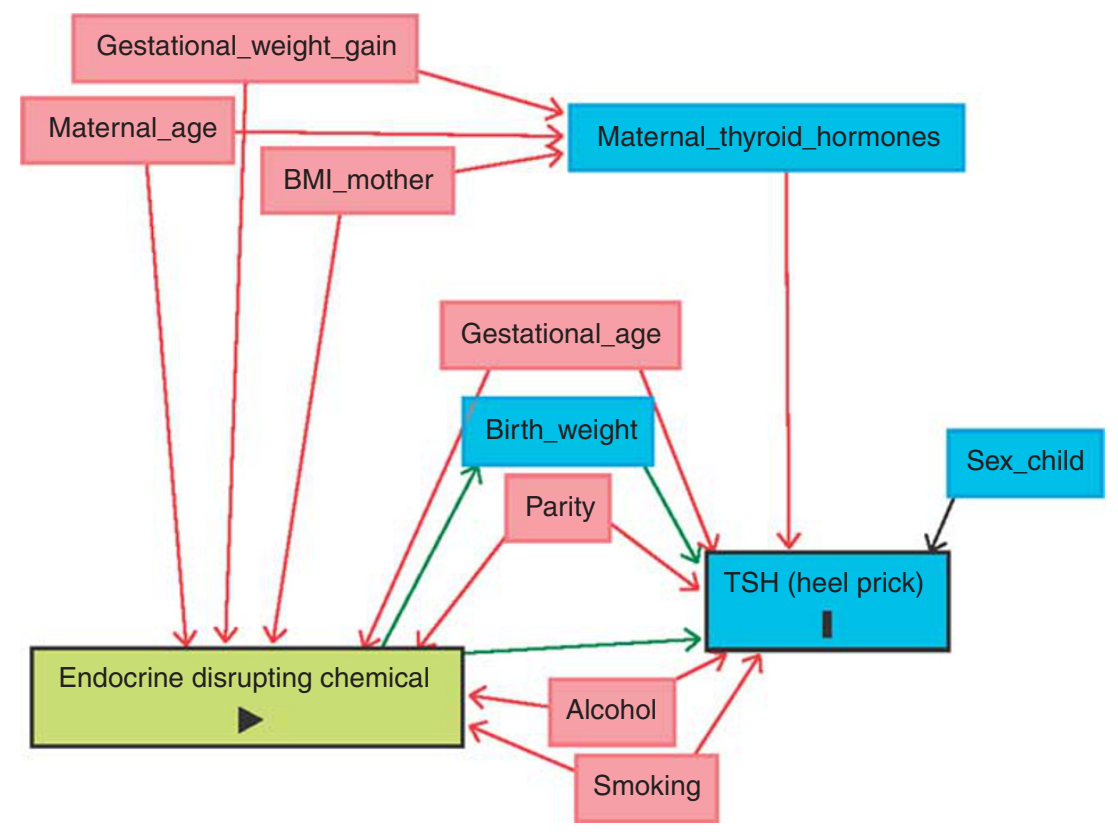

Figure 1. Directed acyclic graph for potential covariates in the association between exposure to endocrine-disrupting chemicals (EDCs) and thyroid stimulating hormone (TSH) levels in heel prick blood spots. Variables in red are confounders. Covariates were selected on the basis of the literature, for which references can be found in Supplementary Material S3.

Breast milk in the PCB cohort was collected at day 14 after delivery. Samples were analyzed for PCB-153, p, $\mathrm{p}^{\prime}$-DDE, HCB, PFOS, PFOA, BDE-47, BDE-99, and HBCD. Data on PCB-153, p,p'-DDE, and $\mathrm{HCB}$ were available on all three cohorts, whereas the other compounds were only determined in HUMIS and the PCB cohort. More information on chemical analytical measurements is given in Supplementary Material S2.

\section{Covariates}

Information on the sex and birth weight of the child, weight and height of the mother, and maternal GWG was extracted from medical records of the children or from their mothers. Gestational age was calculated on the basis of ultrasound measurements or from the first day of the last menstrual cycle, or by a combination of both methods. Questionnaires were used to collect data on the mother's birth date, parity, maternal smoking during pregnancy (yes/no), and alcohol intake during pregnancy (drinks per week).

\section{Data Analysis}

Data analysis was performed using SPSS version 21 (IBM, Armonk, NY). Exposures below the LOQ were replaced by LOQ $/ \sqrt{ } 2$. Similar to previous studies on other European cohorts, conversion factors were used to transform levels in breast milk to levels in cord blood (27). The following conversion factors were used:

[PCB-153, cord plasma $(\mathrm{ng} / \mathrm{l})]=1.20 \times[\mathrm{PCB}-153$, breast milk (ng/g lipid)] (27)

$\left[\mathrm{p}, \mathrm{p}^{\prime}-\mathrm{DDE}\right.$, cord plasma $\left.(\mathrm{ng} / \mathrm{l})\right]=1.20 \times\left[\mathrm{p}, \mathrm{p}^{\prime}-\mathrm{DDE}\right.$, breast milk (ng/g lipid)] (27)

[HCB, cord plasma $(\mathrm{ng} / \mathrm{l})=2.15 \times[\mathrm{HCB}$, breast milk (ng/g lipid) $]$ (Murinova et al., unpublished)

As the method to measure TSH was different in Slovakia compared to that in other cohorts, we tested for interaction between cohorts. None of the interaction terms reached statistical significance $(P>0.10)$; therefore, we continued with pooled analysis. For each compound, we fitted multiple regression models with exposure and cohort as fixed factors. Distribution of TSH was checked for normality and outliers using histograms. Owing to the skewed distributions of TSH for all cohorts, we applied a natural log transformation. Furthermore, there was no linear relation between
TSH and the majority of compounds; therefore, we categorized each exposure into quartiles on the basis of pooled data of all cohorts and included these as dummy variables in the models.

Covariates were selected using a Directed Acyclic Graph (Figure 1; Supplementary Material S3). For continuous covariates, the association with TSH was checked and covariates were included in the models regardless of the degree of confounding of the association between exposure to compounds and TSH. As birth weight is considered to be associated with maternal thyroid hormones, TSH and EDCs, we created an adjusted model with and without this variable. Gestational weight gain was added to the models as well, as there are indications that this is an important confounder. However, it is not clear how this may differ for lipophilic and non-lipophilic compounds; therefore we added this parameter to the models separately.

Multiple imputation was used to account for missing data (5.64\%). The imputation model included all the variables included in the models. As data was mainly missing for gestational weight gain (39.3\%) and alcohol consumption (6.1\%), we also included education in the imputation model.

For each model, we plotted and checked the residuals for normal distribution. We assessed effect modification by sex and smoking, and performed stratified analyses if the $P$-value of the interaction term was $P<0.10$.

\section{RESULTS}

The cohorts were similar for most characteristics (Table 1). In FLEHSI, a higher percentage of participants were nulliparous, whereas mothers were on average younger in the PCB cohort, and HUMIS children were heavier at birth. When compared on basic characteristics, excluded mother-child pairs were similar to those included, except regarding birth weight and smoking. Excluded children from the FLEHSI cohort had a significantly lower birth weight than did included children from this cohort $(P<0.05)$, whereas excluded mothers from HUMIS tended to smoke more than those included $(P<0.05)$. 


\section{Articles $\mid$ de cock et al.}

TSH levels were comparable for FLEHSI and HUMIS, whereas median TSH in the PCB cohort was considerably higher (median TSH, respectively, 1, 1.10, and $2.76 \mathrm{mU} / \mathrm{l}$ ) (Table 2). This was similar for the P90, which was higher in the PCB cohort. The percentage of samples < limit of quantification (LOQ) was 46.1 and $22.9 \%$ for FLEHSI and HUMIS, respectively.

Median levels of PCB-153, p, p'-DDE, and HCB were higher in the $\mathrm{PCB}$ cohort compared with those in the other two cohorts (Table 3). Exposure to PFOA was comparable in HUMIS and the PCB cohort. For PFOS, however, median exposure levels were higher in HUMIS (median PFOS HUMIS: $75.5 \mathrm{ng} / \mathrm{l}$ vs. PCB cohort: $32 \mathrm{ng} / \mathrm{l}$ ). All three flame retardants were detectable at very low levels in HUMIS in the majority of participants, but relatively high levels were observed in some samples. For example, the median BDE-47 level was $0.96 \mathrm{ng} / \mathrm{g}$ lipid, with a minimum exposure level of $0.15 \mathrm{ng} / \mathrm{g}$ lipid, whereas the maximum exposure level was $55.76 \mathrm{ng} / \mathrm{g}$ lipid.

We observed an overall significant negative association between exposure to PCB-153 in quartiles and TSH levels in heel prick blood spots for both the crude and adjusted models $(P=0.018$ and $P=0.07-0.008$, respectively) (Table 4). Including birth weight and gestational weight gain in the models did not affect the results. Also for $\mathrm{p}, \mathrm{p}^{\prime}-\mathrm{DDE}$ an overall negative association between exposure and TSH was observed, which reached statistical significance after adjustment (crude: $P=0.059$; adjusted: $P=0.037-0.040$ ). TSH was not statistically significantly associated with any of the other compounds. We observed no interaction with either sex or smoking.

For PCB-153 exposure, the only significant association with $\mathrm{TSH}$ concentration was in the third exposure quartile as compared with the first quartile of exposure. In the adjusted model, the ratio of the geometric mean of TSH in the third quartile compared to that in the first quartile of exposure was 0.86 (95\% confidence interval (CI): 0.76-0.96), indicating that these children had a $14 \%$ (95\% CI: 4-24\%) lower TSH level at birth. Also for $\mathrm{p}, \mathrm{p}^{\prime}$-DDE, a lower TSH level was observed in the third quartile, a difference that was significant across all models (ratio adjusted GM Q3 vs. Q1 =0.85; 95\% CI: 0.75-0.96).

\section{DISCUSSION}

The objective of this study was to study associations between early life exposure to selected EDCs and TSH levels in heel prick blood spots in three European birth cohorts. For two of the cohorts, FLEHSI and HUMIS, TSH levels were comparable; however, the median TSH level in the PCB cohort was considerably higher. In the largest study to date on EDCs and $\mathrm{TSH}$, we found higher PCB-153 and p,p'-DDE levels to be associated with a decrease in TSH levels in newborns, and associations appeared to be non-linear.

Overall significant associations between PCB-153 exposure and TSH levels were observed. The results are similar to what Dallaire et al. (17) had observed in plasma samples from 7-month-old Inuit infants, as well as in cord blood samples from infants from the Nunavik region in Canada (28).
Table 2. TSH levels $(\mathrm{mU} / \mathrm{l})$ in heel prick blood spots for each cohort

\begin{tabular}{lrccccc}
\hline & $n$ & Min & P10 & Median & P90 & Max \\
\hline FLEHSI & 1,105 & <LOD & <LOD & 1 & 3 & 8.80 \\
HUMIS & 230 & $<$ LOD & <LOD & 1.10 & 2.71 & 17.30 \\
PCB cohort & 449 & 0.23 & 1.36 & 2.76 & 5.22 & 16.82 \\
\hline
\end{tabular}

However, in both studies, associations were attenuated after adjustment. Most studies to date have not observed any association between TSH levels in newborns and exposure to PCB-153 (22,29), whereas one reported a positive association (18). However, these studies measured TSH levels in cord or infant blood, collected shortly after birth, which may not be the ideal matrix as TSH levels may have been elevated because of delivery. Only one small study $(n=23)$ measured TSH levels in heel prick blood spots and found no association with PCB-153 exposure (23).

We also observed significantly lower TSH plasma levels within the third quartile of $\mathrm{p}, \mathrm{p}^{\prime}$-DDE exposure. Ribas-Fito et al. (30) previously published a negative association between p,p'-DDE and TSH in cord blood (30), in line with our findings. However, other studies are scarce and mostly report null associations. Associations between p, $\mathrm{p}^{\prime}$-DDE and TSH remained relatively stable after adjustment for gestational weight gain (GWG), though not for all imputed data sets. We included GWG in our models as a potential confounder because maternal weight gain dilutes the concentrations of toxicants (31), and is related to both maternal TSH and fT4 (32).

To date, most studies on BDEs and TSH have reported no associations $(21,22,33)$, although some studies, such as the one by Shy et al. (21), do report associations with other thyroid hormones. However, similar to the studies on PCB-153, TSH was mostly measured in cord or infant blood, collected shortly after birth, which may have biased their results. We found no associations between BDE-47, BDE-99, and $\mathrm{HBCD}$ and $\mathrm{TSH}$, in line with previous studies. Also for HCB, we observed no effect on TSH. Two previous studies, however, reported negative associations $(19,34)$. In one of those studies, exposure levels were considerably lower than that in our cohorts (34), whereas in the other study exposure was determined in the placenta, complicating comparability of exposure levels between studies (19).

We observed no associations between PFOS, PFOA, and TSH. Only one study thus far has investigated PFOS or PFOA in relation to TSH levels in newborns (35). However, no association was observed, although a positive increase in TT4 was seen. In addition, other observational studies have reported effects of PFAS exposure on thyroid hormones, both in the fetus and in the adult $(36,37)$. As experimental studies have shown that these compounds may displace thyroid hormones from transport proteins such as albumin and transthyretin, it is plausible that PFAAs interact with thyroid hormone homeostasis (38); however, the findings of the current study do not support this assumption. 
Table 3. Exposure profiles per cohort as well as for all cohorts pooled

\begin{tabular}{|c|c|c|c|c|c|c|}
\hline Compound & Matrix & $n$ & Median & Range & LOQ & $<$ LOQ (\%) \\
\hline \multicolumn{7}{|l|}{ PCB-153 (ng/l) } \\
\hline FLEHSI & Cord & 1,020 & 60 & $14.14-450$ & 20 & 19.6 \\
\hline HUMIS converted (ng/l) & Cord (converted) & 230 & 39.04 & $8.81-115.61$ & & \\
\hline PCB cohort & Cord & 405 & 311.48 & $6.23-6,388.55$ & $3.38-15.49$ & 0.5 \\
\hline \multicolumn{7}{|l|}{$p, p^{\prime}-D D E(n g / l)$} \\
\hline FLEHSI & Cord & 1,065 & 220 & $14.14-3,740$ & 20 & 1.7 \\
\hline HUMIS (ng/g lipid) & Milk & 230 & 41.07 & $6-384.69$ & 0.224 & 0 \\
\hline HUMIS converted (ng/l) & Cord (converted) & 230 & 49.28 & $7.20-461.63$ & & \\
\hline \multicolumn{7}{|l|}{$\mathrm{HCB}(\mathrm{ng} / \mathrm{l})$} \\
\hline FLEHSI & Cord & 999 & 40 & $14.14-320$ & 20 & 24.6 \\
\hline HUMIS (ng/g lipid) & Milk & 230 & 10.96 & $4.34-41.97$ & 0.1338 & 0 \\
\hline HUMIS converted (ng/l) & Cord (converted) & 230 & 23.56 & $9.33-90.24$ & & \\
\hline PCB cohort & Cord & 399 & 189.28 & $2.87-2,967.58$ & $1.41-57.92$ & 6.8 \\
\hline Pooled & Cord & 1,628 & 44 & $2.87-2,967.58$ & & 16.8 \\
\hline \multicolumn{7}{|l|}{ PFOS (ng/l) } \\
\hline HUMIS & Milk & 82 & 75.50 & $32-320$ & $4.8-17$ & 0 \\
\hline Pooled & Milk & 194 & 27 & $5.50-210$ & & 12.4 \\
\hline \multicolumn{7}{|l|}{ BDE-47 (ng/g lipid) } \\
\hline HUMIS & Milk & 230 & 0.96 & $0.15-55.76$ & 0.02 & 0 \\
\hline PCB cohort & Milk & 114 & 0.30 & $0.12-3.30$ & $0.1-2.5$ & 66.7 \\
\hline Pooled & Milk & 344 & 0.74 & $0.12-55.76$ & & 22.1 \\
\hline \multicolumn{7}{|l|}{ BDE-99 (ng/g lipid) } \\
\hline HUMIS & Milk & 230 & 0.26 & $0.02-9.52$ & 0.02 & 0 \\
\hline PCB cohort & Milk & 114 & 0.12 & $0.05-1.20$ & $0.07-1.3$ & 86.8 \\
\hline Pooled & Milk & 344 & 0.21 & $0.02-9.52$ & & 28.8 \\
\hline \multicolumn{7}{|l|}{ HBCD (ng/g lipid) } \\
\hline HUMIS & Milk & 186 & 0.54 & $0.13-31.20$ & 0.2 & 32.3 \\
\hline PCB cohort & Milk & 114 & 0.83 & $0.23-24$ & $0.23-5.3$ & 50 \\
\hline Pooled & Milk & 300 & 0.67 & $0.13-31.20$ & & 39 \\
\hline
\end{tabular}




\section{Articles | de Cock et al.}

Table 4. Results of the regression models for TSH levels (mU/l) per compound (in quartiles) in newborns from three European mother-child cohorts

PCB-153 (ng/l) (F+H+P)

\begin{tabular}{|c|c|c|c|c|c|c|c|c|c|}
\hline & \multirow[t]{2}{*}{$n$} & \multirow[t]{2}{*}{ Q1 (<36.78) } & \multicolumn{2}{|c|}{ Q2 (36.78-70) } & \multicolumn{2}{|c|}{ Q3 (70-160) } & \multicolumn{2}{|c|}{$\mathrm{Q} 4(\geqslant 160)$} & \multirow[t]{2}{*}{ Overall $P$-value } \\
\hline & & & GM & $95 \% \mathrm{Cl}$ & GM & $95 \% \mathrm{Cl}$ & GM & $95 \% \mathrm{Cl}$ & \\
\hline Crude & 1,654 & Ref & 0.97 & $0.872-1.069$ & 0.88 & $0.791-0.979$ & 1.06 & $0.914-1.231$ & 0.018 \\
\hline Adjusted $^{\mathrm{b}}$ & 1,583 & Ref & 0.96 & $0.912-1.016$ & 0.86 & $0.809-0.909$ & 1.05 & $0.892-1.229$ & $0.007-0.008$ \\
\hline+ Birth weight $^{c}$ & 1,583 & Ref & 0.96 & $0.914-1.018$ & 0.86 & $0.810-0.911$ & 1.04 & $0.890-1.226$ & $0.009-0.010$ \\
\hline$+\mathrm{GWG}^{\mathrm{d}}$ & 1,579 & Ref & 0.97 & $0.868-1.075$ & 0.86 & $0.763-0.963$ & 1.04 & $0.885-1.224$ & $0.007-0.012$ \\
\hline \multicolumn{10}{|c|}{$\mathrm{p}, \mathrm{p}^{\prime}-\mathrm{DDE}(\mathrm{ng} / \mathrm{l})(\mathrm{F}+\mathrm{H}+\mathrm{P})$} \\
\hline & \multirow[t]{2}{*}{$n$} & \multirow[t]{2}{*}{ Q1 $(<108.43)$} & \multicolumn{2}{|c|}{ Q2 (108.43-239.99) } & \multicolumn{2}{|c|}{ Q3 (240-574.49) } & \multicolumn{2}{|c|}{ Q4 ( $\geqslant 574.49)$} & Overall $P$-value \\
\hline & & & & $95 \% \mathrm{Cl}$ & GM & $95 \% \mathrm{Cl}$ & GM & $95 \% \mathrm{Cl}$ & \\
\hline Crude & 1,700 & Ref & 0.92 & $0.819-1.030$ & 0.86 & $0.765-0.970$ & 0.96 & $0.841-1.107$ & 0.059 \\
\hline Adjusted $^{b}$ & 1,627 & Ref & 0.90 & $0.797-1.007$ & 0.85 & $0.754-0.957$ & 0.96 & $0.887-1.029$ & $0.037-0.040$ \\
\hline+ Birth weight $^{\mathrm{c}}$ & 1,627 & Ref & 0.90 & $0.798-1.010$ & 0.85 & $0.757-0.955$ & 0.96 & $0.887-1.029$ & $0.040-0.043$ \\
\hline$+\mathrm{GWG}^{\mathrm{d}}$ & 1,622 & Ref & 0.90 & $0.796-1.012$ & 0.85 & $0.750-0.961$ & 0.95 & $0.822-1.103$ & $0.018-0.053$ \\
\hline \multicolumn{10}{|c|}{$\mathrm{HCB}(\mathrm{ng} / \mathrm{l})(\mathrm{F}+\mathrm{H}+\mathrm{P})$} \\
\hline & \multirow[t]{2}{*}{$n$} & \multirow[t]{2}{*}{ Q1 (<16.77) } & \multicolumn{2}{|c|}{ Q2 (16.77-39.99) } & \multicolumn{2}{|c|}{ Q3 (40-88.51) } & \multicolumn{2}{|c|}{$\mathrm{Q} 4(\geqslant 88.52)$} & Overall $P$-value \\
\hline & & & GM & $95 \% \mathrm{Cl}$ & GM & $95 \% \mathrm{Cl}$ & GM & $95 \% \mathrm{Cl}$ & \\
\hline Crude & 1,628 & Ref & 1.09 & $0.986-1.215$ & 0.98 & $0.885-1.094$ & 1.06 & $0.932-1.200$ & 0.184 \\
\hline Adjusted $^{b}$ & 1,558 & Ref & 1.07 & $0.969-1.183$ & 0.97 & $0.886-1.052$ & 1.05 & $0.982-1.127$ & $0.251-0.287$ \\
\hline+ Birth weight $^{c}$ & 1,558 & Ref & 1.07 & $1.033-1.112$ & 0.97 & $0.876-1.080$ & 1.06 & $0.986-1.132$ & $0.277-0.305$ \\
\hline$+\mathrm{GWG}^{\mathrm{d}}$ & 1,554 & Ref & 1.07 & $0.965-1.196$ & 0.97 & $0.871-1.085$ & 1.06 & $0.922-1.211$ & $0.138-0.328$ \\
\hline \multicolumn{10}{|l|}{ PFOS (ng/l) $(\mathrm{H}+\mathrm{P})$} \\
\hline & \multirow[t]{2}{*}{$n$} & \multirow[t]{2}{*}{ Q1 $(<29)$} & \multicolumn{2}{|c|}{ Q2 (29-45.99) } & \multicolumn{2}{|c|}{ Q3 (46-79.99) } & \multicolumn{2}{|c|}{$\mathrm{Q} 4(\geqslant 80)$} & Overall $P$-value \\
\hline & & & GM & $95 \% \mathrm{Cl}$ & GM & $95 \% \mathrm{Cl}$ & GM & $95 \% \mathrm{Cl}$ & \\
\hline Crude & 193 & Ref & 1.12 & $0.827-1.511$ & 1.08 & $0.750-1.543$ & 1.14 & $0.785-1.650$ & 0.877 \\
\hline Adjusted $^{b}$ & 185 & Ref & 1.03 & $0.752-1.421$ & 1.03 & $0.702-1.514$ & 1.03 & $0.674-1.562$ & $0.805-0.998$ \\
\hline+ Birth weight ${ }^{\mathrm{C}}$ & 185 & Ref & 1.01 & $0.732-1.395$ & 1.04 & $0.707-1.535$ & 1.01 & $0.659-1.536$ & $0.935-0.999$ \\
\hline$+\mathrm{GWG}^{\mathrm{d}}$ & 185 & Ref & 0.99 & $0.712-1.389$ & 1.03 & $0.691-1.524$ & 0.97 & $0.625-1.505$ & $0.762-0.998$ \\
\hline
\end{tabular}

PFOA (ng/l) $(\mathrm{H}+\mathrm{P})$

$$
n \quad \mathrm{Q} 1(<16)
$$

Crude

Adjusted $^{\mathrm{b}}$

+ Birth weight $^{c}$

+ GWG $^{\mathrm{d}}$

BDE-47 (ng/g lipid) (H+P)

Crude
Adjusted $^{\mathrm{b}}$
+ Birth weight $^{\mathrm{c}}$
+ GWG $^{\mathrm{d}}$

194

186

186

186

$n \quad$ Q1 $(<0.35)$

341

327

327

327

\section{Ref}

Ref

Ref

Ref

Q1 $(<0.35)$

Ref

Ref

Ref

Ref

Q2
GM
1.04
1.06
1.06

Q2 (16-26.99)

GM $\quad 95 \% \mathrm{Cl}$

Q3 (27-40.99)

0.773-1.410

0.767-1.462

0.776-1.488

$0.757-1.473$

$\begin{array}{cc}\text { Q2 } & (0.35-0.73) \\ \mathrm{GM} & 95 \% \mathrm{Cl}\end{array}$

1.13

0.872-1.457

1.11

0.839-1.473
$1.10 \quad 0.829-1.456$

$1.11 \quad 0.836-1.471$

Q3
GM
1.08
1.03
1.02
1

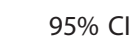

0.805-1.446

$0.749-1.426$

$0.738-1.418$

$0.714-1.401$

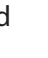


BDE-99 (ng/g lipid) (H+P)

\begin{tabular}{|c|c|c|c|c|c|c|c|c|c|}
\hline \multirow{3}{*}{ 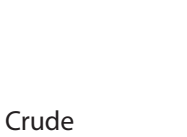 } & \multirow{2}{*}{$n$} & \multirow[t]{2}{*}{ Q1 $(<0.12)$} & \multicolumn{2}{|c|}{ Q2 (0.12-0.20) } & \multicolumn{2}{|c|}{ Q3 (0.21-0.33) } & \multicolumn{2}{|c|}{$\mathrm{Q} 4(\geqslant 0.34)$} & \multirow[t]{2}{*}{ Overall $P$-value } \\
\hline & & & GM & $95 \% \mathrm{Cl}$ & GM & $95 \% \mathrm{Cl}$ & GM & $95 \% \mathrm{Cl}$ & \\
\hline & 330 & Ref & 1.01 & $0.785-1.296$ & 0.92 & $0.703-1.213$ & 1.02 & $0.776-1.337$ & 0.858 \\
\hline Adjusted $^{\mathrm{b}}$ & 317 & Ref & 1.02 & $0.787-1.334$ & 0.92 & $0.692-1.223$ & 1.03 & $0.771-1.363$ & $0.815-0.842$ \\
\hline+ Birth weight $^{c}$ & 317 & Ref & 1.02 & $0.782-1.327$ & 0.90 & $0.680-1.203$ & 1.03 & $0.772-1.364$ & $0.750-0.779$ \\
\hline$+\mathrm{GWG}^{\mathrm{d}}$ & 317 & Ref & 1.02 & $0.785-1.332$ & 0.91 & $0.681-1.210$ & 1.02 & $0.765-1.354$ & $0.763-0.826$ \\
\hline
\end{tabular}

$\mathrm{HBCD}(\mathrm{ng} / \mathrm{g}$ lipid) $(\mathrm{H}+\mathrm{P})$

\begin{tabular}{|c|c|c|c|c|c|c|c|c|c|}
\hline & \multirow[t]{2}{*}{$n$} & \multirow[t]{2}{*}{ Q1 $(<0.31)$} & \multicolumn{2}{|c|}{ Q2 (0.31-0.66) } & \multicolumn{2}{|c|}{ Q3 (0.67-1.20) } & \multicolumn{2}{|c|}{$\mathrm{Q} 4(\geqslant 1.21)$} & \multirow[t]{2}{*}{ Overall $P$-value } \\
\hline & & & GM & $95 \% \mathrm{Cl}$ & GM & $95 \% \mathrm{Cl}$ & GM & $95 \% \mathrm{Cl}$ & \\
\hline Crude & 300 & Ref & 0.98 & $0.755-1.280$ & 0.99 & $0.765-1.284$ & 1.11 & $0.848-1.447$ & 0.772 \\
\hline Adjusted $^{\mathrm{b}}$ & 286 & Ref & 1 & $0.756-1.320$ & 1.01 & $0.764-1.327$ & 1.12 & $0.841-1.490$ & $0.793-0.818$ \\
\hline+ Birth weight $^{c}$ & 286 & Ref & 1 & $0.758-1.325$ & 0.99 & $0.754-1.306$ & 1.11 & $0.835-1.486$ & $0.804-0.825$ \\
\hline$+\mathrm{GWG}^{\mathrm{d}}$ & 286 & Ref & 1 & $0.756-1.320$ & 1 & $0.761-1.327$ & 1.09 & $0.850-1.458$ & $0.872-0.920$ \\
\hline
\end{tabular}

F, FLEHSI; GM, ratio of geometric mean compared to reference; $H, H U M I S ; P, P C B$ cohort.

aRange of $P$-values for the imputed datasets.

${ }^{\text {b} M o d e l ~ a d j u s t e d ~ f o r: ~ c o h o r t, ~ s e x, ~ g e s t a t i o n a l ~ a g e, ~ m a t e r n a l ~ a g e ~ a t ~ b i r t h, ~ p a r i t y, ~ s m o k i n g, ~ m a t e r n a l ~ B M I . ~}$

${ }^{c}$ Adjusted model+birth weight.

${ }^{\mathrm{d}}$ Adjusted model+birth weight+GWG.

Value indicates the ratio of the TSH level in a specific quartile for a specific model, compared to the lowest quartile (percentage).

We did not observe effect modification by sex, in line with previous studies. No sex-specific standards are used for the heel prick screening of thyroid disorders. However, thyroid disease occurs more frequently in women, and several studies have shown that there are sex-specific aspects to the regulation of thyroid hormones. Thyrocytes, for example, are known to have androgen and estrogen receptors (39), and both androgens and estrogens may influence the growth and differentiation of these cells (40), which is modulated through TSH (41). Future studies should keep this in mind and be aware of possible sex-specific effects.

This study is unique in that it investigated a large variety of compounds across multiple cohorts. Variability in exposure levels was high, particularly for PCB-153, p,p'-DDE, and HCB, for which children in FLEHSI and HUMIS had relatively low exposure levels, similar to various other European cohorts (27), whereas children from the PCB cohort had exposure levels similar to that found in Inuit populations (42). This range in exposure levels has given more insight into dose-response relations, which were nonlinear in this study. Nonmonotonic dose-response curves are not unexpected, given the endocrine-disrupting properties of the chemicals (16). However, to accurately assess doseresponse relations, replication from other studies with a similar dose range is required, these studies should include non-linear models. Furthermore, the health effects observed may be dependent on genetic factors, as well as on the presence of other chemicals. In this paper, we assessed exposure compound-by-compound, leaving mixture effects unaddressed. Although new methods for analyses are discussed, there is currently no clear strategy for analyzing these effects. One should bear in mind that the compound-bycompound approach may select highly collinear toxicants and does not address the additive or otherwise synergistic effects of the actual total mix of toxicants in the body.

A strength of this study is that neonatal screening for thyroid disorders is usually performed $4-6$ days after birth. As TSH levels stabilize during the first 2 days after birth (43), and sampling occurred after this period, we did not have to consider factors that are often expected to influence TSH levels in cord blood, such as mode of delivery (44). Moreover, we were able to pool the FLEHSI, HUMIS, and PCB cohorts, resulting in the largest study published thus far on this topic. However, for the purpose of pooling, conversion factors were used, which may have introduced error and therefore may have attenuated associations. Furthermore, measurement of covariates may have differed per cohort, and as we could only use data that were available for all cohorts we have to consider the possibility of residual confounding. No corrections for multiple testing were applied, and spurious associations cannot be completely excluded. Although for some compounds the sample size was large, for the majority of compounds only data from a relatively small group of participants were available.

Indications of disruption of thyroid hormones by environmental chemicals have been mainly based on animal studies, except for PCB-153. For other chemicals, observational studies have been scarce and results are not convincing. 
Flame retardants were considered plausible candidates for thyroid hormone disruption in animal studies, but this has not been supported by data from human studies. With regard to PCB-153 and p,p'-DDE, previous studies thus far have not found convincing results for an association with TSH. We could only observe significant associations for PCB-153 in the imputed data set. These associations were robust and remained statistically significant after adjustment for confounders. This study therefore indicates PCB-153 and $\mathrm{p}, \mathrm{p}^{\prime}-\mathrm{DDE}$ as potential disruptors of TSH.

\section{CONCLUSIONS}

In the largest study to date, we found an association between early life exposure to PCB-153, p, $\mathrm{p}^{\prime}$-DDE, and newborn TSH levels in three European cohorts. Higher exposure levels were associated with $12-15 \%$ lower TSH levels. Associations were quite robust for both PCB-153 and p,p'-DDE. Future studies should assess TSH after birth when it has stabilized and should not assume a linear association. Whether these lower TSH levels pose a risk to the infants requires substantiation from other studies.

\section{SUPPLEMENTARY MATERIAL}

Supplementary material is linked to the online version of the paper at http://www.nature.com/pr

\section{ACKNOWLEDGMENTS}

We would like to thank all the families who participated in the cohort studies. Without them, this research would not have been possible. Furthermore, we would like to extend our gratitude to Irva Hertz-Picciotto, who, as one of the principal investigators of the PCB cohort, enabled this study.

\section{STATEMENT OF FINANCIAL SUPPORT}

This research was funded by the European Community's Seventh Framework Program [FP7/2007-2013] under Grant Agreement OBELIX No. 227391.

Disclosure: The authors declare no conflict of interest.

\section{REFERENCES}

1. CDC Fourth Report on Human Exposure to Environmental Chemicals. Atlanta, GA: U.S. Department of Health and Human Services, Centers for Disease Control and Prevention, 2009.

2. Woodruff TJ, Zota AR, Schwartz JM. Environmental chemicals in pregnant women in the United States: NHANES 2003-2004. Environ Health Perspect 2011;119:878-5.

3. Valvi D, Mendez MA, Martinez D, et al. Prenatal concentrations of polychlorinated biphenyls, DDE, and DDT and overweight in children: a prospective birth cohort study. Environ Health Perspect 2011;120:451-7.

4. Luzardo OP, Mahtani V, Troyano JM, et al. Determinants of organochlorine levels detectable in the amniotic fluid of women from Tenerife Island (Canary Islands, Spain). Environ Res 2009;109:607-13.

5. Wigle DT, Arbuckle TE, Turner MC, et al. Epidemiologic evidence of relationships between reproductive and child health outcomes and environmental chemical contaminants. J Toxicol Environ Health B Crit Rev 2008;11:373-517.

6. Iszatt N, Stigum H, Verner MA, et al. Prenatal and postnatal exposure to persistent organic pollutants and infant growth: a pooled analysis of seven European birth cohorts. Environ Health Perspect 2015;123:730-6.
7. Smink A, Ribas-Fito N, Garcia R, et al. Exposure to hexachlorobenzene during pregnancy increases the risk of overweight in children aged 6 years. Acta Pædiatr 2008;97:1465-9.

8. Iszatt N, Stigum H, Govarts E, et al. Perinatal exposure to dioxins and dioxin-like compounds and infant growth and body mass index at seven years: a pooled analysis of three European birth cohorts. Environ Int 2016;94:399-407.

9. Halldorsson TI, Rytter D, Haug LS, et al. Prenatal exposure to perfluorooctanoate and risk of overweight at 20 years of age: a prospective cohort study. Environ 2012;120:668-73.

10. Forns J, Mandal S, Iszatt N, et al. Novel application of statistical methods for analysis of multiple toxicants identifies DDT as a risk factor for early child behavioral problems. Environ Res 2016;151:91-100.

11. Newbold RR. Developmental exposure to endocrine-disrupting chemicals programs for reproductive tract alterations and obesity later in life. Am J Clin Nutr 2011;94:16.

12. Shi X, Liu C, Wu G, Zhou B. Waterborne exposure to PFOS causes disruption of the hypothalamus-pituitary-thyroid axis in zebrafish larvae. Chemosphere 2009;77:1010-8.

13. Obregon MJ, Calvo RM, Del Rey FE, de Escobar GM. Ontogenesis of thyroid function and interactions with maternal function. Endocr Dev 2007;10:86-98.

14. Bernal J. Thyroid hormones in brain development and function. In: De Groot LJ, Beck-Peccoz P, Chrousos G et al., eds. Endotext. South Dartmouth, MA: MDText.com Inc, 2000.

15. Meerts IA, Lilienthal H, Hoving S, et al. Developmental exposure to 4-hydroxy-2,3,3',4',5-pentachlorobiphenyl (4-OH-CB107): long-term effects on brain development, behavior, and brain stem auditory evoked potentials in rats. Toxicol Sci 2004;82:207-18.

16. Vandenberg LN, Colborn T, Hayes TB, et al. Hormones and endocrinedisrupting chemicals: low-dose effects and nonmonotonic dose responses. Endocr Rev 2012;33:378-455.

17. Dallaire R, Muckle G, Dewailly E, et al. Thyroid hormone levels of pregnant inuit women and their infants exposed to environmental contaminants. Environ Health Perspect 2009;117:1014-20.

18. Alvarez-Pedrerol M, Ribas-Fito N, Torrent M, et al. Thyroid disruption at birth due to prenatal exposure to beta-hexachlorocyclohexane. Environ Int 2008;34:737-40.

19. Freire C, Lopez-Espinosa MJ, Fernandez M, Molina-Molina JM, Prada R, Olea N. Prenatal exposure to organochlorine pesticides and TSH status in newborns from Southern Spain. Sci Total Environ 2011;409: 3281-7.

20. Lin SM, Chen FA, Huang YF, et al. Negative associations between PBDE levels and thyroid hormones in cord blood. Int J Hyg Environ Health 2011;214:115-20.

21. Shy CG, Huang HL, Chao HR, Chang-Chien GP. Cord blood levels of thyroid hormones and IGF-1 weakly correlate with breast milk levels of PBDEs in Taiwan. Int 2012;215:345-51.

22. Herbstman JB, Sjodin A, Apelberg BJ, et al. Birth delivery mode modifies the associations between prenatal polychlorinated biphenyl (PCB) and polybrominated diphenyl ether (PBDE) and neonatal thyroid hormone levels. Environ Health Perspect 2008;116:1376-82.

23. Otake T, Yoshinaga J, Enomoto T, et al. Thyroid hormone status of newborns in relation to in utero exposure to PCBs and hydroxylated PCB metabolites. Environ Res 2007;105:240-6.

24. Tehrani RF, Aghaee M, Asefzadeh S. The comparison of thyroid function tests in cord blood following cesarean section or vaginal delivery. Int J Endocrin Metab 2003;1:22-6.

25. Legler J, Hamers T, van Eck van der Sluijs-van de Bor M, et al. The OBELIX project: early life exposure to endocrine disruptors and obesity. Am J Clin Nutr 2011;94:1933S-8S.

26. PerkinElmer. Congenital Hypothyroidism Screening, 2007. (http://www. perkinelmer.com/CMSResources/Images/44-72928BRO_ CongenitalHypothyroidism.pdf.). Accessed 17 February 2014.

27. Govarts E, Nieuwenhuijsen M, Schoeters G, et al. Birth weight and prenatal exposure to polychlorinated biphenyls (PCBs) and dichlorodip- 
henyldichloroethylene (DDE): a meta-analysis within 12 European birth cohorts. Environ Health Persp 2012;120:162-70.

28. Dallaire R, Dewailly E, Ayotte P, Muckle G, Laliberte C, Bruneau S. Effects of prenatal exposure to organochlorines on thyroid hormone status in newborns from two remote coastal regions in Quebec, Canada. Environ Res 2008;108:387-92.

29. Maervoet J, Vermeir G, Covaci A, et al. Association of thyroid hormone concentrations with levels of organochlorine compounds in cord blood of neonates. Environ Health Perspect 2007;115:1780-6.

30. Ribas-Fito N, Sala M, Cardo E, et al. Organochlorine compounds and concentrations of thyroid stimulating hormone in newborns. Occup Environ Med 2003;60:301-3.

31. Verner MA, Sonneborn D, Lancz K, et al. Toxicokinetic modeling of persistent organic pollutant levels in blood from birth to 45 months of age in longitudinal birth cohort studies. Environ Health Perspect 2013;121: $131-7$.

32. Pop VJ, Biondi B, Wijnen HA, Kuppens SM, Lvader H. Maternal thyroid parameters, body mass index and subsequent weight gain during pregnancy in healthy euthyroid women. Clin Endocrinol 2013;2013: 12177.

33. Eggesbo M, Thomsen C, Jorgensen JV, Becher G, Odland JO, Longnecker MP.. Associations between brominated flame retardants in human milk and thyroid-stimulating hormone (TSH) in neonates. Environ Res 2011:111:737-43.

34. Li C, Cheng Y, Tang Q, et al. The association between prenatal exposure to organochlorine pesticides and thyroid hormone levels in newborns in Yancheng, China. Environ Res 2014;129:47-51.

35. Lopez-Espinosa MJ, Mondal D, Armstrong B, Bloom MS, Fletcher T. Thyroid function and perfluoroalkyl acids in children living near a chemical plant. Environ Health Perspect 2012;120:1036-41.
36. Kim S, Choi K, Ji K, et al. Trans-placental transfer of thirteen perfluorinated compounds and relations with fetal thyroid hormones. Environ Sci Technol 2011;45:7465-2.

37. Knox SS, Jackson T, Frisbee SJ, Javins B, Ducatman AM. Perfluorocarbon exposure, gender and thyroid function in the C8 Health Project. J Toxicol Sci 2011;36:403-10.

38. Luo Z, Shi X, Hu Q, Zhao B, Huang M. Structural evidence of perfluorooctane sulfonate transport by human serum albumin. Chem Res Toxicol 2012;25:990-2.

39. Banu SK, Govindarajulu P, Aruldhas MM. Testosterone and estradiol upregulate androgen and estrogen receptors in immature and adult rat thyroid glands in vivo. Steroids 2002;67:1007-4.

40. Stanley JA, Aruldhas MM, Yuvaraju PB, et al. Is gender difference in postnatal thyroid growth associated with specific expression patterns of androgen and estrogen receptors? Steroids 2010;75:1058-66.

41. Banu KS, Aruldhas MM. Sex steroids regulate TSH-induced thyroid growth during sexual maturation in Wistar rats. Exp Clin Endocrinol Diabet 2002;110:37-42.

42. Cartier C, Muckle G, Jacobson SW, et al. Prenatal and 5-year p,p'-DDE exposures are associated with altered sensory processing in school-aged children in Nunavik: a visual evoked potential study. Neurotoxicology 2014;44c:8-16.

43. Fisher DA, Klein AH. Thyroid development and disorders of thyroid function in the newborn. N Engl J Med 1981;304:702-12.

44. Herbstman J, Apelberg BJ, Witter FR, Panny S, Goldman LR. Maternal, infant, and delivery factors associated with neonatal thyroid hormone status. Thyroid 2008;18:67-76. 